\title{
A Limit on Galactic Extinction Not Correlated with Far IR Emission
}

\author{
Douglas P. Finkbeiner ${ }^{1}$, David J. Schlegel ${ }^{2}$, and Marc Davis ${ }^{1}$
}

1 University of California at Berkeley, Departments of Physics and Astronomy, 601 Campbell Hall, Berkeley, CA 94720

2 University of Durham, Department of Physics, South Road, Durham DH1 3LE, United Kingdom

\begin{abstract}
We have reprocessed the IRAS and DIRBE full-sky $100 \mu \mathrm{m}$ maps, using HI as a zero-point, to produce a map of Galactic extinction. This map is crosscorrelated with APM galaxy counts over the full APM region, including dusty regions of the sky not used to compute the APM correlation function in Maddox et al.(1990a). The angular correlation function, $w(\theta)$, of galaxy counts is computed with and without the extinction correction, and is used to suggest that our map accounts for $\sim 90 \%$ of the dust-induced correlation in the APM region on scales from $2-10^{\circ}$. This is the first time that a limit has been placed on the correlation function of the extinction not represented in a given map.
\end{abstract}

\section{Introduction}

The Burstein \& Heiles $(1978,1982)$ reddening map has been the standard method of estimating Galactic extinction for nearly 20 years. At the meeting, I presented an alternative method based on $100-240 \mu \mathrm{m}$ emission from dust, using $\mathrm{HI}$ emission for a zero-point. The calibration from infrared emission to $A(B)$ extinction is based on the $\mathrm{Mg}_{2}$ - color relation for elliptical galaxies (Faber et al.1989), and will be reported in Schlegel et al.(1998).

Because dust affects galaxy counts in a non-trivial way (Heiles) we use a likelihood analysis to determine the right coefficient for correcting APM galaxy counts. We will use this, along with information about the underlying correlation of the APM counts, to put a limit on how much extinction is not detected by our map. In addition to placing limits on the interstellar medium within our Galaxy, such a limit is critical for studying the largescale structure in the universe. In addition, we claim that the limit applies to any dust associated with a layer of HII in our Galaxy.

\section{Data}

The SFD Map: In Schlegel et al.(1998) we will present a reprocessing of the IRAS and DIRBE all-sky 100-240 $\mu \mathrm{m}$ maps, which trace the interstellar medium. We have improved the ISSA maps (Wheelock et al.1994) with a 
Fourier destriping method, and have removed 20,000 confirmed point sources. Spatial scales larger than 1 degree have been replaced with data from DIRBE $\left(0.7^{\circ}\right.$ beam $)$ using an appropriate scaling. The DIRBE $25 \mu \mathrm{m}$ map is used as a spatial template for zodiacal light, but is modulated in a 3 parameter model that minimizes dependence of the dust-gas residual upon ecliptic latitude. The $100-240 \mu \mathrm{m}$ channels of the DIRBE maps are used to recover the temperature of the dust, and convert the emission to column density. The composite map is smoothed to a $\sim 6^{\prime}$ roughly-Gaussian beam, and is known as the Schlegel-Finkbeiner-Davis (SFD) map.

APM Galaxy Survey: The APM galaxy survey (Maddox et al.1990b,c) covers a large region of the southern sky at high Galactic latitudes, and provides a background distribution of galaxy counts that are extinguished by dust in our Galaxy. The dust extinction, measured by the SFD maps, can be calibrated by studying the statistical covariance between the APM and SFD maps: dusty regions have increased dust emission and diminished galaxy counts. The large number of galaxies in the APM survey $\left(2.8 \times 10^{6}\right)$ provides excellent statistics.

\section{Likelihood Analysis}

The calibration of extinction in the APM galaxy maps is very amenable to a maximum-likelihood solution. In this analysis, we treat the underlying mean galaxy counts, $\bar{N}$, the extinction normalization, $p$, and the star leakage parameter, $q$, as model parameters.

Each APM pixel, or cell, has an expected number of galaxies drawn from. the underlying galaxy distribution, modified by the extinction in that cell. In addition, each cell contains a number of stars which have been confused with galaxies - this usually happens because two stars are very near each other. We compute the likelihood of measuring the observed number of galaxies, $N_{i}$ in cell $i$ for a grid of star and dust models. The likelihood of a model is the product of the likelihoods of each independent cell. We choose the model with the highest likelihood.

The underlying galaxy distribution is well sampled in the high Galactic latitudes of the APM maps. The area at $|b|>50^{\circ}$ is a "clean" region largely free of Galactic dust contamination. The observed distribution of galaxies in the clean APM region is well fit by a "counts in cells" function (Saslaw \& Hamilton 1984) which gives $f\left(N_{i} \mid \bar{N}\right)$ where $N_{i}$ is the observed number of counts in cell $i$, and $\bar{N}$ is the mean number of counts per cell of the entire APM region. This function is similar to a Poisson distribution, but takes the spatial correlation of galaxies into account.

Because dust will reduce the number of counts in a cell, we now define the expected mean number of counts in a cell with dust to be 


$$
\bar{N}^{\prime}=\bar{N} 10^{-\gamma g p I_{100}}
$$

where $g$ is a geometrical/completeness factor ( $g=0.6$ for Euclidean space), $p$ is a ratio of $100 \mu \mathrm{m}$ flux to $A(B), I_{100}$ is $100 \mu \mathrm{m}$ flux, and $\gamma$ is a parameter of order unity that depends on the specifics of the APM survey. In these terms, $A(B)=p I_{100}$.

For point sources, $\gamma=1$, but there are several effects that can cause $\gamma$ to deviate from unity for galaxies. Extinction changes the size of a galaxy's isophotes, and may cause galaxies to be confused with stars more easily at the faint end of the survey. Also, the non-linearities of photographic film can cause the behavior of galaxies to differ from that of stars. See Heiles (1976) and Santiago et al.(1996) for a discussion of this effect.

Because of these ambiguities, the APM analysis alone cannot break the degeneracy between $p, g$, and $\gamma$. The product $\gamma g p$ that we fit in the likelihood analysis is appropriate for the APM survey, but lacking a separate measurement of $g$ and $\gamma$, this is not a measurement of $p$ or $A(B)$.

\section{Correlation Function}

A main motivation for the APM survey was to measure the angular correlation function, $w(\theta)$. We display $w(\theta)$ in Figure 1a, calculated for the following cases: uncorrected counts $\left(w_{\text {raw }}\right)$, corrected counts $\left(w_{\text {corr }}\right)$, clean counts $\left(w_{\text {clean }}\right)$, and dust $\left(w_{\text {dust }}\right)$. The "clean" counts are counts in a region where our dust correction has little effect. Also displayed in Figure 1a is $w_{d u s t}+w_{\text {clean }}$. Correlation functions obey a simple addition rule, $w_{A \times B}=$ $w_{A}+w_{B}$ for two maps, $A$ and $B$. Therefore, the extinction not accounted for by the SFD map is $w_{\text {? }}=w_{\text {corr }}-w_{\text {clean }}$. We call this the "excess correlation" in the corrected map, and compare it to the "excess correlation," $w_{\text {raw }}-w_{\text {clean }}$, in the raw map in Figure $1 \mathrm{~b}$.

\section{Conclusions}

We have placed a constraint on the spatial correlation of extinction not present in the SFD map. Such extinction must be either small or uniform. If it is uniform, it may be constrained by stellar reddening measurements, as in Schlegel et al.(1998). Although we have attempted to correct for dust temperature, our assumptions may break down near shock fronts, GMCs, and other regions of unusual temperature. However, at high latitude, the SFD map appears to account for $\sim 90 \%$ of the observed extinction (See Fig. 1b). This limit includes extinction from dust uncorrelated with $\mathrm{Hr}$ such as dust in the Reynolds layer.

Acknowledgements. The COBE data sets were developed by the NASA Goddard Space Flight Center under the guidance of the COBE Science Working Group 

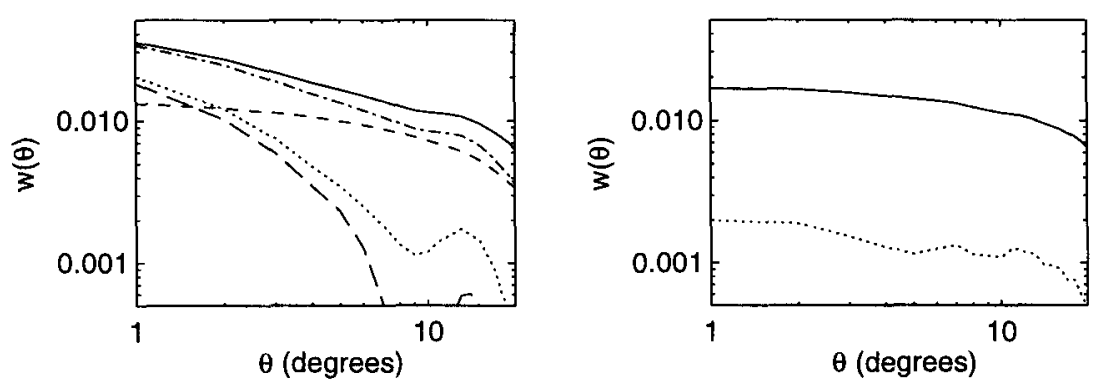

Fig. 1. The solid line in Fig. 1a is the correlation function of the entire APM region. The upper dashed line is dust, and the lower dashed line is the clean APM region (corrected), taken to be the underlying galaxy correlation. The dotted line is the correlation of the full APM region after correcting for dust and stars. The dash-dot line is the sum of the dust and clean lines. In Fig. 1b, the solid and dotted lines are the excess correlation of uncorrected and corrected counts, respectively.

and were provided by the NSSDC. We wish to thank Sherry Wheelock of IPAC for providing ISSA coverage maps, the joint IRAS Science Working Group for all IRAS data, Steve Maddox for APM maps, and Carl Heiles for invaluable discussions and guidance. DF acknowledges support from an NSF Graduate Fellowship. This work was supported by NASA grant NAG5-1360.

\section{References}

Burstein, D. \& Heiles, C. 1978, ApJ, 225, 40

Burstein, D. \& Heiles, C. 1982, AJ, 87, 1165

Maddox, S.J., et al.1990a, MNRAS, 242, 43p

Maddox, S.J., et al.1990b, MNRAS, 243, 692

Maddox, S.J., Efstathiou, G., \& Sutherland, W.J. 1990c, MNRAS, 246, 433

Hartmann, D. \& Burton, W.B. 1996, Atlas of Galactic Neutral Hydrogen, Cambridge University Press.

Faber S.M., et al.1989, ApJS, 69, 763

Heiles, C. 1976, ApJ, 204, 379

Santiago, B.X. 1996, et al.ApJ, 461, 38

Saslaw, W.C. \& Hamilton, A.J.S. 1984, ApJ, 276, 13

Schlegel, D. J., Finkbeiner, D. P. and Davis, Marc 1998 in prep

Wheelock, S.L. et al.1994, IRAS Sky Survey Atlas: Explanatory Supplement, JPL Publication 94-11 (Pasadena: JPL) 